\title{
Atitudes e práticas dos médicos de família do ACeS de Matosinhos face à obesidade
}

\author{
Ângela Neves*
}

\section{RESUMO}

Objetivos: Caracterizar as atitudes e práticas dos médicos de família do Agrupamento de Centros de Saúde (ACeS) de Matosinhos relativamente à obesidade e aos pacientes obesos. Tipo de estudo: Transversal.

Local: ACeS de Matosinhos, Portugal.

População: Médicos especialistas e internos de formação específica de medicina geral e familiar (MGF) do ACeS de Matosinhos Métodos: Foi utilizada uma amostra de conveniência constituída por todos os médicos presentes na reunião semanal da respetiva unidade funcional. Como instrumento de recolha de dados foi desenvolvido um questionário de autopreenchimento, onde se incluíram seis dimensões avaliadas: «autoeficácia e competência técnica», «carências formativas», «convicções», «responsabilidade autopercecionada», «barreiras» e «limitações». Na análise de dados procedeu-se ao estudo de validade e fiabilidade das dimensões, bem como da construção de variáveis observadas para as mesmas, a partir da sua média (M), enquadrando-as numa escala de Likert (1-5). Para medir as diferenças entre os grupos foram utilizadas estatísticas não-paramétricas.

Resultados: Dos 104 participantes, a maioria (71\%) não realizou formação pós-graduada na área da nutrição/alimentação. Porém, reconhecem a necessidade de aprofundar conhecimentos na área da nutrição/alimentação, com uma média de 3,96 na dimensão das carências formativas percecionadas. Os médicos inquiridos demonstraram um elevado nível de convicção $(M=4,33)$ acerca da importância do seu papel no tratamento da obesidade, reconhecendo firmemente a sua responsabilidade $(M=4,27)$ no tratamento desta patologia, com perceção de autoeficácia e competência técnica na área $(M=3,58)$. Quanto às limitações e barreiras sentidas, os resultados sugerem que estes profissionais reconhecem algumas limitações e barreiras no tratamento da obesidade $(M=3,29$ e $M=2,60$, respetivamente).

Conclusões: Os médicos de família demonstram atitudes e práticas globalmente positivas face à obesidade e aos pacientes obesos. Estes profissionais estão convictos acerca da importância do seu papel na gestão/tratamento da obesidade, sentem-se responsáveis pelo tratamento desta patologia e reconhecem a sua necessidade formativa na área da nutrição/alimentação.

Palavras-chave: Obesidade; Médicos de família; Atitudes; Práticas.

\section{INTRODUÇÃO}

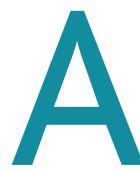
obesidade é definida pela Organização Mundial da Saúde (OMS) como uma anormal ou excessiva acumulação de gordura corporal que pode atingir graus capazes de afetar a saúde. ${ }^{1}$

A crescente epidemia de obesidade constitui um sério problema de saúde pública nos países desenvolvidos. No ano de 2008, a OMS estimava a existência de cerca de 1,5 mil milhões de adultos com excesso de peso, sendo que $500 \mathrm{mi}$ lhões se encontravam na faixa da obesidade, projetando-se, para o ano de 2015, um acréscimo para os 2,3 mil milhões de adultos com excesso de peso e 700 milhões de obesos. ${ }^{1}$

Em Portugal, o estudo Alimentação e Estilo deVida da População Portuguesa, promovido pela Sociedade Portugue-

*Médica interna de Medicina Geral e Familiar. ULS de Matosinhos - USF Lagoa sa de Ciências de Nutrição e Alimentação, em 2009, mostrou que, dos 3.529 participantes, $40 \%$ apresentava excesso de peso e $10,8 \%$ obesidade, com uma prevalência total de obesidade/excesso de peso de $50,8 \%$, sendo as prevalências de excesso de peso mais elevadas nas regiões dos Açores (71,3\%), Madeira (59,7\%) e Alentejo (57,5\%). ${ }^{2}$

As expectativas futuras não afiguram resultados otimistas, atendendo que dados recentes sugerem a existência de cerca de 3,5 milhões de portugueses a sofrer de excesso de peso e um milhão de obesidade. ${ }^{3}$

Apesar da clara evidência científica acerca dos benefícios da redução de peso, sabe-se que os resultados obtidos encontram-se muito aquém dos resultados desejáveis e metas delineadas em cuidados de saúde primários (CSP).4-5

A eficácia das intervenções comunitárias e programas de gestão do peso, baseadas em programas comportamen- 
tais, alimentares (restrição calórica) e exercício físico (incremento de atividade física), em doentes com excesso ponderal têm-se revelado pouco eficazes na perda ponderal e manutenção de um peso saudável, sem o impacto clinicamente desejável na luta contra a obesidade. ${ }^{6-9}$

Não obstante, as diferentes justificações para este insucesso como a falta de envolvimento e motivação dos pacientes obesos, o escasso tempo de consulta e a inexistência de metas/indicadores e programas governamentais específicos como os que existem para outras doenças crónicas em particular, entre outras, ${ }^{10}$ diversos estudos apontam os médicos de família (MF) como possíveis responsáveis pelos resultados insatisfatórios obtidos neste âmbito. ${ }^{5,11-12}$

Conhecimentos e formação insuficientes, ${ }^{14-15}$ conceções desajustadas e estereótipos acerca dos pacientes obesos, baixa perceção sobre a capacidade e motivação destes pacientes para incitar mudanças, sentimentos débeis de autoeficácia e de competência técnica para gerir e tratar o excesso de peso/obesidade, depreciação de uma prática clínica preventiva, tempo de consulta exíguo e falta de remuneração e de incentivos a uma prática preventiva são alguns dos aspetos apontados por diversos estudos que se debruçam sobre as diferentes barreiras e posturas negativas dos MF na gestão e tratamento da obesidade como doença crónica que é..$^{13,16-17}$

De uma forma genérica, os MF reconhecem a obesidade como sendo um problema de saúde significativo e que justifica ser abordado. No entanto, nem todos admitem dispor de responsabilidade e competências para a sua abordagem, depositando a responsabilidade do desenvolvimento e tratamento desta patologia aos próprios pacientes obesos. ${ }^{17-18}$

Por isso, revela-se de extrema importância conhecer as principais atitudes e práticas dos MF portugueses em relação aos pacientes obesos, de forma a delinear estratégias dirigidas e individualizadas de sensibilização destes profissionais acerca do modo como as suas crenças e atitudes poderão condicionar a sua prática clínica, bem como o seu relacionamento com estes doentes e a sua adesão ao regime terapêutico, com vista à prestação de cuidados de saúde de excelência. ${ }^{19-20}$

O Agrupamento de Centros de Saúde (ACeS) de Matosinhos integra a Unidade Local de Saúde (ULS) de Matosinhos, uma instituição pública empresarial, integrada no Serviço Nacional de Saúde. O seu serviço de nutrição disponibiliza uma consulta de nutrição, realizada nos quatro centros de saúde do concelho, em quatro períodos semanais, mediante pedido formulado pelo MF, de acordo com indi- cadores pré-definidos; bem como diferentes projetos de âmbito escolar, comunitário, hospitalar e formativo, nomeadamente para o desenvolvimento de competências específicas para médicos e enfermeiros de família, na área do aconselhamento alimentar, com vista a otimizar o papel destes profissionais de saúde como agentes promotores de educação alimentar. O projeto "Formação sobre Alimentação Saudável para Médicos e Enfermeiros de Família”, em curso na ULSM desde 2012, constitui um exemplo da formação contínua e capacitação interdisciplinar levada a cabo nesta mesma instituição em particular.

Em Portugal não existe, ainda, publicado nenhum estudo desta natureza, pelo que este trabalho teve como objetivo caracterizar as atitudes e práticas dos MF do ACeS de Matosinhos relativamente à obesidade e aos pacientes obesos.

\section{MÉTODOS}

Realizou-se um estudo transversal, tendo a recolha de dados decorrido entre setembro de 2014 e fevereiro de 2015.

A população em estudo correspondeu aos médicos especialistas em medicina geral e familiar (MGF) e internos de formação específica de MGF a desempenhar funções nas Unidades de Saúde Familiar (USF) e Unidades de Cuidados de Saúde Personalizados (UCSP) do ACeS de Matosinhos (149 profissionais), tendo-se utilizado uma amostra de conveniência, constituída pelos 104 médicos respondentes ao questionário que se encontravam presentes na reunião semanal de serviço da sua respetiva unidade funcional.

A realização deste estudo foi aprovada pela Comissão de Ética da ULS de Matosinhos, após submissão do respetivo protocolo.

Os dados foram colhidos através de um questionário elaborado pela investigadora, com base na revisão da literatura nacional e internacional, ${ }^{12,15-16,21-23}$ a fim de possibilitar a comparação de resultados. A primeira parte foi composta por questões dirigidas à caracterização sociodemográfica e profissional dos inquiridos e a segunda parte por dezoito afirmações/itens, distribuídos por seis subescalas que avaliam as atitudes e práticas dos MF face à obesidade: «autoeficácia e competência técnica» avaliada por cinco itens; «carências formativas percecionadas» por dois itens; «convicções» por três itens; «responsabilidade autopercecionada» por três itens; «barreiras» por três itens e «limitações» por dois itens; os itens foram classificados numa escala tipo Likert: 1 - Discordo absolutamente, 2 - Discordo, 3-Não concordo, nem discordo, 4-Concordo e 5-Concordo absolutamente; a cada item foi atribuída uma pon- 
tuação entre 1-5; as afirmações negativas foram codificadas inversamente (Anexo I).

A pontuação das dimensões analisadas foi dividida em três segmentos, de acordo com a amplitude da escala de Likert utilizada: baixo [1-2,33], médio [2,33-3,66] e elevado [3,66-5].

Estes seis aspetos correspondem a dimensões distintas mas relacionadas com as atitudes e práticas face à obesidade, sendo que atitudes e práticas positivas face à obesidade refletem-se em valores elevados nas subescalas «autoeficácia e competência técnica», «convicções» e «responsabilidade autopercecionada» e baixos nas subescalas «carências formativas percecionadas», «barreiras» $\mathrm{e}$ «limitações»; atitudes e práticas neutras são traduzidas por valores médios nos scores das seis subescalas; atitudes e práticas negativas face à obesidade refletem-se em valores baixos na "autoeficácia e competência técnica», «convicções» e «responsabilidade autopercecionada» e elevados nas subescalas "carências formativas percecionadas", «barreiras»e «limitações».

Este questionário foi submetido a um teste-piloto aplicado a cinco dos profissionais do universo em estudo, o que permitiu avaliar os vários aspetos metodológicos e aperfeiçoar a técnica de recolha de dados, tendo-se excluído os profissionais que nele participaram.

Como variáveis independentes foram consideradas: género, idade, funções, ano de conclusão da sua formação pré-graduada, habilitações académicas, formação pós-graduada em nutrição/alimentação, principais fontes de informação, número de horas de formação despendidas nos últimos três anos e tempo dedicado em cada consulta a conselhos sobre alimentação e atividade física.

As variáveis dependentes consideradas foram atribuídas às dimensões:

- Autoeficácia e competência técnica: autoeficácia e competência técnica na gestão/tratamento da obesidade (cinco itens);

- Carências formativas percecionadas: carências formativas percecionadas na área da nutrição/alimentação (dois itens);

- Convicções: convicções acerca da importância do seu papel na gestão/tratamento da obesidade (três itens);

- Responsabilidade autopercecionada: responsabilidade sentida no tratamento da obesidade (três itens);

- Barreiras: barreiras sentidas no tratamento da obesidade (três itens);

- Limitações: limitações percecionadas na gestão/tratamento da obesidade (dois itens).
O referido questionário, anónimo e de autopreenchimento, foi entregue a cada um dos profissionais de saúde no dia da reunião da sua unidade, após o contacto prévio com o respetivo coordenador. Após a sua execução, cada profissional depositava o seu questionário no interior de uma urna, reservada para esse efeito, de forma a garantir o anonimato.

A análise de dados foi realizada com recurso ao programa software Statistical Package for the Social Sciences, v. 21,0®. Nas análises exploratórias foram calculadas médias (M), desvios-padrão (DP), frequências absolutas ( $n$ ) e relativas (\%). Os itens com formulação contrária à dos restantes itens de cada dimensão foram revertidos. A fiabilidade e validade das dimensões do questionário foram medidas, respetivamente, com o alfa de Cronbach $(>0,60),{ }^{24}$ correlação item-total $(>0,30)^{25}$ e com uma análise em componentes principais (ACP). ${ }^{26}$ Foram avaliados os pressupostos Kaiser-Meyer-Olkin (KMO $>0,60)^{25}$ e o teste de esfericidade de Bartlett (BTS $p<0,001) .{ }^{26}$ Considera-se expectável que a variância explicada seja superior a $50 \%$ e a carga fatorial de cada item seja superior a $0,45 .{ }^{27}$

Cada uma das dimensões foi posteriormente construída com base na média dos itens que as compunham, mantendo assim a sua estrutura inicial, com uma escala variável entre 1 (discordo absolutamente) e 5 (concordo absolutamente). De seguida, estas variáveis foram avaliadas quando à normalidade da sua distribuição, com o teste Kolmogorov-Smirnov, utilizado nos casos em que a dimensão amostral é superior a 50 elementos..$^{25}$

Atendendo a que nem todas as dimensões calculadas obtiveram distribuição normal, foram utilizadas estatísticas não-paramétricas para medir as diferenças entre grupos, utilizando para isso o teste Mann-Whitney para comparação de grupos e o coeficiente de correlação de Spearman para o estabelecimento de correlações (rs).

Para cada uma das dimensões foi implementado um modelo de regressão linear, tendo em conta apenas as variáveis cuja correlação foi estatisticamente significativa. Nestes modelos foram medidos os coeficientes e respetivos IC 95\%. Foram também verificados e cumpridos os pressupostos de normalidade de resíduos (Kolmogorov-Smirnov, $p>, 05$ ) e outliers para o critério $r_{i}<|3|$. A inexistência de autocorrelação dos resíduos foi analisada e verificada com o teste Durbin Watson. Nos modelos com mais do que uma variável independente foi ainda verificada a multicolinariedade recorrendo aos critérios de tolerância $>, 10$ e VIF $<4$.

Todas as análises tiveram em conta a rejeição da hipótese nula baseada no critério da significância estatística (5\%). 


\section{RESULTADOS}

A amostra do presente estudo foi composta por 104 médicos maioritariamente do sexo feminino (79\%), com idades compreendidas entre os 25 e os 61 anos $(M=37,7)$. Cerca de $55 \%$ eram licenciados e $45 \%$ possuíam grau de mestre, sendo que nenhum dos participantes detinha um nível académico superior a mestrado. A maioria dos inquiridos era médico especialista em MGF (58\%), sendo os restantes internos de formação específica desta mesma especialidade (42\%). A experiência profissional dos inquiridos variou entre o início de carreira (zero anos) e 37 anos de experiência $(M=13,2)$.

Quando inquiridos relativamente à formação pós-graduada, a maioria dos participantes não dispunha de formação pós-graduada na área da nutrição/alimentação (71\%), sendo que, desses, $33 \%$ apontaram como principal motivo a inexistência de oportunidades formativas na área. Foram também apontados outros motivos, como desconhecimento de ações de formação nesta área (20\%), não ser esta uma área prioritária para as suas funções (17\%), a escassa oferta formativa (16\%) e a falta de tempo (13\%).

Mais de metade dos médicos (52\%) entende que os congressos, workshops e cursos são a fonte privilegiada para recolha de informação na área da nutrição. Outras fontes de informação consideradas foram as revistas científicas (18\%) e a Internet (14\%). Para grande parte dos médicos, o tempo gasto com formação na área da nutrição não excedeu as 20 horas (93\%).

Os médicos referiram despender, em média, 5,6 minutos do tempo total da consulta no aconselhamento dos doentes sobre alimentação e atividade física.

\section{Análises de fiabilidade e validade}

Com as análises de fiabilidade e validade pretendeu-se consolidar a construção teórica do questionário e as dimensões a ele inerentes, através de métodos estatísticos apropriados. ${ }^{30}$

Foram avaliadas as propriedades psicométricas das diferentes dimensões: «Autoeficácia e competência técnica», «Carências formativas percecionadas», «Convicções», «Responsabilidade autopercecionada», «Barreiras» e «Limitações». Todas as dimensões consideradas obtiveram percentagens de variância explicada acima de $50 \%$, o que advoga em favor da sua unidimensionalidade. Os pressupostos $\mathrm{KMO}(>0,60)$ e BTS $(p<, 001)$ foram cumpridos quase na íntegra, com exceção das dimensões relativas à «Responsabilidade autopercecionada» e «Limitações», onde também se registaram os valores mais baixos de alfa de Cronbach e correlação item total. Nas restantes dimen- sões, os resultados da correlação item-total foram de pelo menos 0,30 e o alfa de Cronbach foi superior a 0,60, sendo a única exceção a dimensão das «Convicções».

Desta forma, considerou-se que o instrumento utilizado apresentou boas propriedades psicométricas.

Seguidamente são apresentados os resultados descritivos das diferentes dimensões, calculados pela média dos itens que as compõem.

\section{Atitudes e práticas}

As convicções dos MF acerca da importância do seu papel na gestão/tratamento da obesidade foi a dimensão que obteve resultados mais elevados, com uma média de 4,33. A responsabilidade autopercecionada no tratamento da obesidade e as carências formativas foram dimensões também consideravelmente bem pontuadas. Por fim, em relação às barreiras sentidas pelos MF no tratamento da obesidade, verificou-se uma média de 2,60, a mais baixa de todas as dimensões. Deste modo, traduzindo os valores descritivos das diferentes dimensões em categorias percentuais, verifica-se que a maioria destes profissionais apresenta um elevado nível de convicção acerca da importância do seu papel na gestão/tratamento da obesidade, bem como ao nível da responsabilidade sentida no tratamento desta patologia. Ao nível das carências formativas na área da nutrição/alimentação, uma grande parte dos inquiridos demonstrou uma perceção elevada das suas necessidades formativas neste âmbito. Quanto às barreiras sentidas no tratamento da obesidade, os participantes revelaram uma perceção média a baixa e ao nível das limitações percecionadas na gestão/tratamento da obesidade foi frequente a perceção média a elevada (Quadro I).

A análise das correlações entre as dimensões avaliadas (Quadro II) mostra que a dimensão autoeficácia e a competência técnica dos inquiridos apresentam uma correlação negativa com as carências formativas percecionadas $(p<0,01)$, barreiras percebidas $(p<0,001)$ e limitações sentidas $(p<0,05)$ e uma correlação positiva com a responsabilidade autopercecionada $(p<0,001)$.

Ao nível das carências formativas percecionadas constata-se uma correlação positiva com as convicções $(p<0,01)$, bem como ao nível das barreiras sentidas $(p<0,01)$.

Relativamente à dimensão convicções dos participantes relativamente à importância do seu papel na gestão e tratamento da obesidade denota-se uma correlação positiva com a dimensão da responsabilidade assumida no tratamento desta doença e, em contrapartida, uma correlação negativa na dimensão das barreiras percebidas no tratamento dos doentes obesos. 


\begin{tabular}{|c|c|c|c|c|}
\hline & $M$ & $\begin{array}{c}\text { Baixo } \\
{[1-2,33]}\end{array}$ & $\begin{array}{c}\text { Médio } \\
{[2,33-3,66]}\end{array}$ & $\begin{array}{c}\text { Elevado } \\
{[3,66-5]}\end{array}$ \\
\hline 1- Autoeficácia e competência técnica & 3,58 & $6(5,8 \%)$ & $46(44,2 \%)$ & $52(50,0 \%)$ \\
\hline 2 - Carências formativas & 3,96 & $3(2,9 \%)$ & $27(26,0 \%)$ & $74(71,2 \%)$ \\
\hline 3- Convicções & 4,33 & $0(0 \%)$ & $7(6,7 \%)$ & $97(93,3 \%)$ \\
\hline 4 - Responsabilidade autopercecionada & 4,27 & $0(0 \%)$ & $8(7,7 \%)$ & $96(92,3 \%)$ \\
\hline 5 - Barreiras & 2,60 & $30(28,8 \%)$ & $62(59,6 \%)$ & $12(11,5 \%)$ \\
\hline 6 - Limitações & 3,29 & $11(10,6 \%)$ & $60(57,7 \%)$ & $33(31,7 \%)$ \\
\hline
\end{tabular}

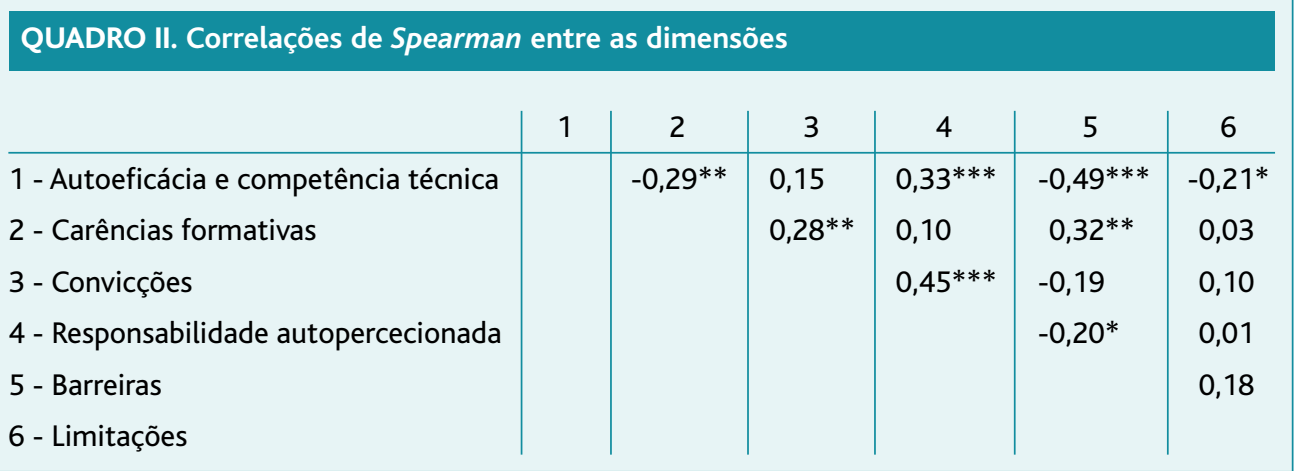

Legenda: ${ }^{*} p<0,05 ;{ }^{* *} p<0,01 ;{ }^{* * *} p<0,001$

Por fim, constata-se, ainda, uma correlação negativa evidente entre a dimensão da responsabilidade assumida pelo tratamento da obesidade e as barreiras sentidas pelos MF que participaram neste estudo.

Relativamente às diferenças por género, a diferença foi estatisticamente significativa apenas na dimensão da autoeficácia e competência técnica $(p=0,03)$, com resultados superiores nos inquiridos do sexo masculino comparativamente com os do sexo feminino (Quadro III).

Quanto às diferenças por funções exercidas, verifica-se que são os internos de formação específica de MGF, em contraste com os especialistas em MGF, os inquiridos que mais reconhecem as suas carências formativas e a necessidade de ampliar conhecimentos na área. Foram também eles os mais convictos a assumir o papel importante do MF na gestão e tratamento da obesidade e os que mais destacam as barreiras existentes no tratamento desta doença (Quadro IV).

Os médicos sem formação pós-graduada na área da nutrição/alimentação foram os inquiridos que mais reconheceram as suas carências formativas na área, bem como os que atribuíram maior relevância às barreiras existentes no tratamento da obesidade. Por outro lado, foram os médicos com formação em nutrição/alimentação os que reportaram uma perceção de maior autoeficácia e competência técnica e maior responsabilidade sentida no tratamento da obesidade (Quadro V).

Os resultados de correlação entre as diferentes dimensões e as variáveis socioprofissionais demonstram que os médicos que despendem mais tempo em formação evidenciam também maior perceção de autoeficácia e competência técnica e uma maior responsabilidade no tratamento da obesidade.

O reconhecimento das carências formativas diminui com a idade e com a experiência profissional. O mesmo aconteceu na relação das convicções acerca da importância do papel do MF na gestão e tratamento da obesidade, com correlações negativas com a idade e experiência profissional.

Por fim, os profissionais que menos limitações apontam no tratamento da obesidade são aqueles quem mais tempo despende, na consulta, a abordar questões na área da nutrição/alimentação e do exercício físico (Quadro VI).

Foram construídos cinco modelos de regressão linear, um por cada dimensão, considerando a existência das correlações mencionadas (Quadro VII). Quadro modelos foram estatisticamente significativos. O tempo em formação tem um impacto positivo e estatisticamente significativo $(\mathrm{p}<0,001)$ na autoeficácia e competência técnica na gestão/tratamento da obesidade e explica $12,50 \%$ desta variável $(p<0,001)$. A experiência profissional mostrou-se estatisticamente significativa em relação às convicções destes profissionais acerca da importância do seu papel na gestão/tratamento da obesidade $(p=0,016)$. Este impacto foi negativo, sugerindo que mais anos de experiência se relacionam com convicções menos fortes $(p<0,05)$.

O tempo em formação apresenta um impacto positivo 
estatisticamente significativo na responsabilidade sentida no tratamento da obesidade $(p=0,016)$, sugerindo que mais formação se relaciona com um maior sentido de responsabilidade $(p<0,001)$.

Por fim, o tempo em consulta a abordar questões de nutrição não apresentou impacto estatisticamente significativo nas limitações percecionadas na gestão/ /tratamento da obesidade.

\section{DISCUSSÃO}

Terminada a análise dos resultados obtidos é possível reconhecer nos MF do ACeS de Matosinhos uma necessidade autopercecionada de formação na área da nutrição/alimentação, sendo que a maioria destes profissionais não realizou formação pósgraduada nesta temática, desfecho que corrobora os estudos descritos anteriormente que avultam a insuficiente formação como uma das numerosas dificuldades na abordagem de pacientes obesos. ${ }^{13-14}$

Os inquiridos reconhecem a necessidade de ampliar conhecimentos e realizar formação na área da alimentação/nutrição e apontam a inexistência de oportunidades de formação e o desconhecimento de ações de formação nesta área como os principais motivos para justificar a escassa formação. Desenlace que vincula os resultados de diversos estudos de investigação que destacam a insatisfação quanto às oportunidades formativas proporcionadas, como sendo uma das barreiras mais citadas à prestação de aconselhamento alimentar. ${ }^{13-14}$

As oportunidades de formação e treino de competências aumentam a autoconfiança e a perceção de autoeficácia, imprescindíveis a uma intervenção eficaz. ${ }^{28}$ Em linha com este relato bibliográfico, foram os inquiridos com formação pós-graduada na área da nutrição/alimentação que reportaram uma maior perceção de autoeficácia e competência técnica na gestão e tratamento da obesidade. ção específica

/alimentação

\begin{tabular}{|c|c|c|c|c|c|}
\hline & \multicolumn{2}{|c|}{$\begin{array}{c}\text { Masculino } \\
(n=22)\end{array}$} & \multicolumn{2}{|c|}{$\begin{array}{c}\text { Feminino } \\
(n=82)\end{array}$} & \multirow[t]{2}{*}{$P$} \\
\hline & M & DP & M & DP & \\
\hline 1 - Autoeficácia e competência técnica & 3,88 & 0,46 & 3,49 & 0,65 & 0,03 \\
\hline 2 - Carências formativas & 3,80 & 0,92 & 4,01 & 0,69 & 0,79 \\
\hline 3 - Convicções & 4,23 & 0,56 & 4,36 & 0,50 & 0,28 \\
\hline 4 - Responsabilidade autopercecionada & 4,35 & 0,42 & 4,25 & 0,51 & 0,55 \\
\hline 5 - Barreiras & 2,65 & 0,61 & 2,59 & 0,77 & 0,36 \\
\hline 6 - Limitações & 3,20 & 0,73 & 3,32 & 0,80 & 0,56 \\
\hline
\end{tabular}

\begin{tabular}{|c|c|c|c|c|c|}
\hline & \multicolumn{2}{|c|}{$\begin{array}{c}\text { ME em MGF } \\
\quad(n=61)\end{array}$} & \multicolumn{2}{|c|}{$\begin{array}{l}\text { IFE de MGF } \\
\quad(n=43)\end{array}$} & \multirow[t]{2}{*}{$P$} \\
\hline & M & DP & M & DP & \\
\hline 1 - Autoeficácia e competência técnica & 3,60 & 0,61 & 3,57 & 0,65 & 0,72 \\
\hline 2 - Carências formativas & 3,73 & 0,73 & 4,26 & 0,65 & $<0,001$ \\
\hline 3 - Convicções & 4,19 & 0,56 & 4,53 & 0,35 & 0,003 \\
\hline 4 - Responsabilidade autopercecionada & 4,21 & 0,55 & 4,36 & 0,40 & 0,28 \\
\hline 5 - Barreiras & 2,44 & 0,74 & 2,79 & 0,69 & 0,01 \\
\hline 6 - Limitações & 3,28 & 0,85 & 3,33 & 0,71 & 0,61 \\
\hline
\end{tabular}

Legenda: ME - Médicos especialista; MGF - Medicina geral e familiar; IFE - Internos de forma-

QUADRO V. Comparação das dimensões segundo formação em nutrição/

\begin{tabular}{|l|c|c|c|c|c|}
\multirow{2}{*}{} & \multicolumn{2}{|c|}{$\begin{array}{c}\text { Sem formação } \\
(n=74)\end{array}$} & $\begin{array}{c}\text { Com formação } \\
(n=30)\end{array}$ & P \\
\cline { 2 - 5 } & $M$ & DP & $M$ & DP & \\
\hline 1 - Autoeficácia e competência técnica & 3,42 & 0,66 & 3,95 & 0,37 & $<0,001$ \\
2 - Carências formativas & 4,07 & 0,71 & 3,68 & 0,77 & 0,03 \\
3 - Convicções & 4,32 & 0,51 & 4,37 & 0,53 & 0,52 \\
4 - Responsabilidade autopercecionada & 4,18 & 0,51 & 4,48 & 0,38 & 0,003 \\
5 - Barreiras & 2,70 & 0,73 & 2,34 & 0,72 & 0,02 \\
6 - Limitações & 3,31 & 0,78 & 3,25 & 0,83 & 0,65 \\
\hline
\end{tabular}

Participantes que expressaram maior autoeficácia e competência na gestão/tratamento da obesidade foram os 


QUADRO VI. Correlações das dimensões com as variáveis socioprofissionais
\begin{tabular}{l|l|l|l|c|} 
& Idade & $\begin{array}{c}\text { Experiência } \\
\text { profissional }\end{array}$ & $\begin{array}{c}\text { Tempo em } \\
\text { formação }\end{array}$ & $\begin{array}{c}\text { Tempo em consulta } \\
\text { a abordar questões } \\
\text { de nutrição }\end{array}$ \\
\hline 1 - Autoeficácia e competência técnica & 0,06 & 0,06 & $0,38^{* * *}$ & 0,04 \\
2 - Carências formativas & $-0,36^{* * *}$ & $-0,39 * * *$ & $-0,15$ & $-0,02$ \\
3 - Convicções & $-0,37^{* * *}$ & $-0,39 * * *$ & 0,19 & $-0,07$ \\
4 - Responsabilidade autopercecionada & $-0,12$ & $-0,17$ & $0,23^{*}$ & 0,07 \\
5 - Barreiras & $-0,16$ & $-0,12$ & $-0,08$ & 0,05 \\
6 - Limitações & $-0,13$ & $-0,15$ & $-0,06$ & $-0,20 *$ \\
\hline
\end{tabular}

Legenda: ${ }^{*} p<0,05 ;{ }^{* * *} p<0,001$

\begin{tabular}{|c|c|c|c|c|c|}
\hline & \multicolumn{3}{|c|}{ Análise dos coeficientes } & \multicolumn{2}{|c|}{ Medidas de ajustamento } \\
\hline & $\square \square$ & $P$ & $95 \%$ IC $\square \square$ & $F_{(103,1)}$ & $\square 2$ \\
\hline \multicolumn{6}{|l|}{ Autoeficácia e competência técnica } \\
\hline$\beta_{0}$ (Constante) & 3,07 & $<0,001$ & $(2,78 ; 3,36)$ & $14,55^{* * *}$ & $12,50 \%$ \\
\hline$\beta_{1}$ Tempo em formação & 0,34 & $<0,001$ & $(0,16 ; 0,52)$ & & \\
\hline \multicolumn{6}{|l|}{ Carências formativas } \\
\hline$\beta_{0}$ (Constante) & 4,12 & $<0,001$ & $(2,72 ; 5,53)$ & $4,47^{*}$ & $8,40 \%$ \\
\hline$\beta_{1}$ Idade & 0,01 & 0,902 & $(-0,05 ; 0,06)$ & & \\
\hline$\beta_{2}$ Experiência profissional & $-0,02$ & 0,421 & $(-0,08 ; 0,03)$ & & \\
\hline \multicolumn{6}{|l|}{ Convicções } \\
\hline$\beta_{0}$ (Constante) & 3,86 & $<0,001$ & $(2,97 ; 4,75)$ & $10,90 * * *$ & $18,40 \%$ \\
\hline$\beta_{1}$ Idade & 0,03 & 0,107 & $(-0,01 ; 0,06)$ & & \\
\hline$\beta_{2}$ Experiência profissional & $-0,05$ & 0,011 & $(-0,08 ;-0,01)$ & & \\
\hline \multicolumn{6}{|l|}{ Responsabilidade autopercecionada } \\
\hline$\beta_{0}$ (Constante) & 4,00 & 0,001 & $(3,77 ; 4,24)$ & $5,99 *$ & $5,60 \%$ \\
\hline$\beta_{1}$ Tempo em formação & 0,18 & 0,016 & $(0,03 ; 0,32)$ & & \\
\hline \multicolumn{6}{|l|}{ Limitações } \\
\hline$\beta_{0}$ (Constante) & 3,51 & 0,001 & $(3,21 ; 3,81)$ & 2,69 & $2,60 \%$ \\
\hline $\begin{array}{l}\beta_{1} \text { Tempo em consulta a abordar } \\
\text { questões de nutrição }\end{array}$ & $-0,04$ & 0,104 & $(-0,10 ; 0,01)$ & & \\
\hline
\end{tabular}

Legenda: ${ }^{*} p<0,05 ;{ }^{* * *} p<0,001$

que evidenciaram menores carências formativas, bem como os que menos valorizam as barreiras e limitações existentes no âmbito do tratamento desta patologia. Foram os profissionais que mais reconhecem a importância do seu papel no tratamento da obesidade os que assumiram uma maior necessidade de ampliar conhecimentos na área, sugerindo que um baixo nível de conhecimento sobre a doença possa associar-se a uma maior valorização das barreiras existentes.

MF convictos relativamente à importância do seu papel na gestão e tratamento da obesidade evidenciam sentir uma maior responsabilidade pelo tratamento desta doença.

De ressaltar, porém, que, independentemente das carências formativas assumidas, bem como das barreiras ou limitações valorizadas, estes profissionais reconhecem o seu papel e responsabilidade no tratamento desta patologia, confirmando os pressupostos apresentados em diferentes estudos que afirmam que os MF reconhecem a obesidade como um problema de saúde significativo e que justifica a sua abordagem. ${ }^{17}$

Vieses involuntários motivados por reação visando prestígio por parte do inquirido, retração defensiva diante de perguntas personalizadas, atração pela resposta positiva, relutância em de- 
clarar limitações, falta de confiança e proficiência no tratamento dos pacientes obesos, perguntas com conteúdo emocional e/ou sentimento de aprovação ou reprovação e indefinição ou ambiguidade nas afirmações poderão ter contribuído como limitações para o presente estudo.

Ainda, como limitações, é de destacar que foram realizados inúmeros testes de inferência estatística sem qualquer ajuste para a erosão do erro alfa, bem como os modelos de regressão obtidos apresentaram um poder explicativo muito baixo (rquadrado sempre inferior a 0,2 ), recomendando-se, nesse sentido, uma análise crítica acerca das associações encontradas como sendo relevantes e verdadeiras.

De realçar que não foram previstas correlações dos $s c o$ res das dimensões com gold standard ou variáveis que, de certa forma, se aproximem dos conceitos das dimensões mencionadas. Não foram, ainda, realizadas análises teste-reteste com aplicação de coeficientes de correlação intraclasse dos scores em dois momentos diferentes para ver a repetibilidade nas dimensões. Porém, estes dois aspetos são mais característicos de um estudo exclusivo e mais exaustivo de validação, que não foi propriamente o objetivo primário do estudo, não se considerando, por isso, fundamentais neste estudo exploratório.

Posto tudo isto, espera-se, assim, que os resultados obtidos neste estudo venham a ter implicações tanto a nível profissional como organizacional, podendo esta ser uma ferramenta de apreciação e consciencialização para o desenvolvimento de futuras estratégias formativas no ACeS de Matosinhos, com vista à promoção de conhecimentos teóricos e competências práticas que colmatem as carências formativas autopercecionadas e promovam a autoeficácia e competência técnica dos MF.

Como proposta de melhoria contínua, realçando a importância deste trabalho pioneiro na área dos CSP e mais propriamente no domínio da MGF, e tratando-se este de um ACeS com características e especificidades próprias de uma ULS, sugere-se uma futura análise comparativa da realidade vivenciada no âmbito das práticas e atitudes dos MF dos diferentes ACeS do nosso país, na perspetiva de um futuro estudo multicêntrico.

Posto tudo isto, conclui-se que os MF do ACeS de Matosinhos demonstram todo um conjunto de atitudes e práticas globalmente positivas face à obesidade e aos pacientes obesos. Estes profissionais revelaram atitudes e práticas convictas acerca da importância do seu papel na gestão/tratamento da obesidade e que atestam a sua responsabilidade percecionada no tratamento desta patologia, nomeadamente ao nível da motivação, aconselha- mento e acompanhamento dos utentes obesos. Assumem-se como profissionais eficazes e competentes que reconhecem a necessidade de uma maior aposta formativa na área da nutrição/alimentação e que, embora percecionando as várias limitações e barreiras existentes no tratamento desta patologia, não descuram a sua função.

\section{REFERÊNCIAS BIBLIOGRÁFICAS}

1. World Health Organization. Obesity and overweight. Geneva:WHO; 2011 [cited 2014 May 5; updated 2016 Jun]. Available from: http://www.who.int/ mediacentre/factsheets/fs311/en/

2. Poínhos R, Franchini B, Afonso C, Correia F, Teixeira VH, Moreira P, et al. Alimentação e estilos de vida da população portuguesa: metodologia e resultados preliminares. Aliment Hum. 2009;15(3):43-60.

3. Direção-Geral da Saúde. Portugal: alimentação saudável em números 2013 [Internet]. Lisboa: DGS; 2013. Available from: https://www.dgs.pt/estatisticas-de-saude/estatisticas-de-saude/publicacoes/portugal-alimentacao-saudavel-em-numeros-2013.aspx

4. Kristeller JL, Hoerr R. Physician attitudes toward managing obesity: differences among six specialty groups. Prev Med. 1997;26(4):542-9.

5. Ogden J, Flanagan Z. Beliefs about the causes and solutions to obesity: a comparison of GPs and lay people. Patient Educ Couns. 2008;71(1):72-8.

6. Centers for Disease Control and Prevention. Losing weight [Internet]. Atlanta: CDC; 2015 [cited 2016 May 4; updated 2015 May 15]. Available from: https://www.cdc.gov/healthyweight/losing_weight/index.html

7. Booth HP, Prevost AT, Gulliford MC. Access to weight reduction interventions for overweight and obese patients in UK primary care: population-based cohort study. BMJ Open. 2015;5(1):e006642.

8. Booth HP, Prevost TA, Wright AJ, Gulliford MC. Effectiveness of behavioural weight loss interventions delivered in a primary care setting: a systematic review and meta-analysis. Fam Pract. 2014;31(6):643-53.

9. Wadden TA, Butryn ML, Hong PS, Tsai AG. Behavioral treatment of obesity in patients encountered in primary care settings: a systematic review. JAMA. 2014;312(17):1779-91.

10. Ogden J. Obesity treatment. In: Ogden J, editor. The psychology of eating: from healthy to disordered behavior: from healthy to disordered behavior. 2nd ed. New York: Wiley-Blackwell; 2010. p. 180-210.

11. Epstein L, Ogden J. A qualitative study of GPs' views of treating obesity. $\mathrm{Br}$ J Gen Pract. 2005;55(519):750-4.

12. Fogelman $Y$, Vinker S, Lachter J, Biderman A, Itzhak B, Kitai E. Managing obesity: a survey of attitudes and practices among Israeli primary care physicians. Int J Obes. 2002;26(10):1393-7.

13. Flores S, García-Lorda P, Munné C, Salas-Salvadó J. La formación en dietética y nutrición vista desde la perspectiva del médico dedicado a la asistencia primaria [Training in dietetics and nutrition from the point of view of the primary care physician]. Nutr Hosp. 2000;15(2):45-50. Spanish

14. Brotons $C$, Ciurana R, Piñeiro R, Kloppe P, Godycki-Cwirko M, Sammut MR. Dietary advice in clinical practice: the views of general practitioners in Europe. Am J Clin Nutr. 2003;77(4 Suppl):1048S-51S.

15. Ferrante JM, Piasecki AK, Ohman-Strickland PA, Crabtree BF. Family physicians' practices and attitudes regarding care of extremely obese patients. Obesity (Silver Spring). 2009;17(9):1710-6.

16. Foster GD, Wadden TA, Makris AP, Davidson D, Sanderson RS, Allison DB, et al. Primary care physicians' attitudes about obesity and its treatment. Obes 
Res. 2003;11(10):1168-77.

17. Frank A. Futility and avoidance: medical professionals in the treatment of obesity. JAMA. 1993;269(16):2132-3.

18. Ogden J, Bandara I, Cohen H, Farmer D, Hardie J, Minas H, et al. General practitioners' and patients' models of obesity: whose problem is it? Patient Educ Couns. 2001;44(3):227-33.

19. National Institute for Health and Clinical Excellence. Obesity: guidance on the prevention, identification, assessment and management of overweight and obesity in adults and children - NICE clinical guidance no. 43 [Internet]. London: NICE; 2006 [cited 2014 May 29]. Available from: https://www.nice.org.uk/guidance/cg43

20. Academy of Medical Royal Colleges. Measuring up: the medical profession's prescription for the Nation's obesity crisis [Internet]. London: AMRC; 2013. Available from: http://www.aomrc.org.uk/wp-content/uploads/ 2016/05/Measuring_Up_0213.pdf

21. Swift JA, Choi E, Puhl RM, Glazebrook C. Talking about obesity with clients: preferred terms and communication styles of UK pre-registration dieticians, doctors and nurses. Patient Educ Couns. 2013;91(2):186-91.

22. Swift JA, Hanlon S, El-Redy L, Puhl RM, Glazebrook C. Weight bias among UK trainee dietitians, doctors, nurses and nutritionists. J Hum Nutr Diet. 2013;26(4):395-402

23. Rego MA. Conhecimentos, atitudes e práticas dos médicos e enfermeiros, dos cuidados de saúde primários, no tratamento da obesidade: existe uma associação com as práticas pessoais de atividade física? [Dissertation]. Lisboa: Escola Nacional de Saúde Pública, Universidade Nova de Lisboa; 2010. Available from: https://run.unl.pt/handle/10362/4298
24. Pestana MH, Gageiro JN. Análise de dados para ciências sociais: a complementaridade do SPSS. $5^{\text {a }}$ ed. Lisboa: Sílabo; 2008. ISBN 9789726184980

25. Reis E. Estatística multivariada aplicada. $2^{\text {a }}$ ed. Lisboa: Sílabo; 2001. ISBN 9726182476

26. Field A. Discovering statistics using SPSS. 2nd ed. London: Sage; 2005. ISBN 0761944524

27. Maroco J. Análise estatística com utilização do SPSS. $2^{\text {a }}$ ed. Lisboa: Sílabo; 2003. ISBN 9726183316

28. Perrin EM, Vann JC, Lazorick S, Ammerman A, Teplin S, Flower K, et al. Bolstering confidence in obesity prevention and treatment counseling for resident and community pediatricians. Patient Educ Couns. 2008;73(2):179-85.

\section{CONFLITO DE INTERESSES}

A autora declara não ter conflitos de interesse.

\section{COMISSÃO DE ÉTICA}

Estudo realizado após parecer favorável da Comissão de Ética para a Saúde da ARS Norte.

\section{ENDEREÇO PARA CORRESPONDÊNCIA}

Ângela Cristina Pinto Neves

R. Fernando Pessoa, $n^{\circ}$ 52, 4435-245 Rio Tinto

E-mail: angela.neves82@gmail.com

Recebido em 26-06-2015

Aceite para publicação em 16-05-2017

\section{ABSTRACT}

\section{ATTITUDES AND PRACTICES OF GENERAL PRACTITIONERS FROM THE MATOSINHOS GROUP OF HEALTH CENTRES REGARDING OBESITY}

Aims: To study attitudes to obesity and obese patients among General Practitioners in the Group of Health Centres of Matosinhos.

Study design: Cross-sectional.

Setting: Group of Health Centres of Matosinhos, Portugal.

Population: Family medicine specialists and trainees in the Matosinhos Group of Health Centres.

Methods: A convenience sample was used, composed of all physicians attending the weekly staff meeting in their respective clinics. A questionnaire was developed for this study composed of six dimensions including: 'self-efficacy and technical competence', 'training needs', 'convictions', 'self-perceived responsibility', 'barriers' and 'limitations'. The validity and reliability of the dimensions were studied. The variables in these dimensions are presented as a mean $(M)$ on a five point Likert scale. Non-parametric statistical analyses were used to measure the differences between the groups.

Results: Among the 104 study participants, most (71\%) did not have post-graduate training in nutrition. However, they recognize the need to increase their knowledge of nutrition, with an average score of 3.96 in the dimension of the training needs perceived. The physicians participating showed a high level of conviction $(M=4.33)$ about the importance of their role in the treatment of obesity, firmly recognizing their responsibility $(M=4.27)$ in the treatment of this condition, perceiving their self-efficacy and technical competence in the area $(M=3.58)$. Concerning the barriers and limitations felt, the results suggest that these professionals recognize some limitations and barriers while treating obesity $(M=3.29$ and $M=2.60$, respectively).

Conclusions: Family medicine specialists have positive attitudes towards managing obesity and obese patients. These professionals are convinced of the importance of their role in management and treatment of obesity. They feel responsible for the treatment of this condition and recognize their training needs in the area of nutrition.

Keywords: Obesity; General practitioners; Attitudes; Practices. 


\section{ANEXO I \\ Questionário}

\section{Atitudes e práticas dos médicos de família do ACeS de Matosinhos face à obesidade}

Assinale com uma cruz $(X)$ o que melhor se adequa à sua situação pessoal:

1. Sexo:

Masculino

Feminino

2. Idade:

anos

3. No presente momento exerce funções de:

Médico de Família

Interno de MGF

4. Ano de conclusão da sua formação pré-graduada:

5. Habilitações académicas:

Licenciatura

Mestrado

Doutoramento

6. Alguma vez teve formação pós-graduada na área da Nutrição/Alimentação?

Não (Avance para a questão 7)

Sim (Avance para a questão 8)

7. Se não teve formação pós-graduada na área da Nutrição/Alimentação, qual o motivo? (assinale a que considerar mais importante)

Não ter oportunidade de formação
Não ter tempo para formação

Não ter conhecimento de ações de formação nesta área

Não ser uma área prioritária para as suas funções

Pouca oferta de formação

8. Qual a sua fonte privilegiada de acesso a informação acerca de Nutrição/Alimentação? (assinale uma única opção, a que considerar mais importante)

Congressos, workshops, cursos

Revistas científicas

Experiência profissional

Outros profissionais especializados nas áreas

Revistas generalistas

Televisão

Internet

Outra. Qual?

9. Indique uma estimativa do número de horas por si despendidas, nos últimos três anos, em cursos, ações de formação e congressos, relacionados com a área da Nutrição/Alimentação?

Menos de 4 horas

De 4 a 20 horas

De 21 a 40 horas

Mais de 40 horas

10. Quando fornece conselhos sobre alimentação e atividade física, quanto tempo, em média, gasta a abordar estas questões na sua consulta?

Minutos 


\begin{tabular}{|c|c|c|c|c|c|c|}
\hline & & 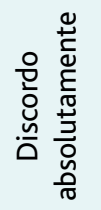 & 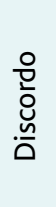 & 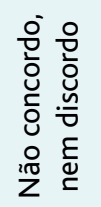 & $\begin{array}{l}\text { 은 } \\
\text { 은 } \\
\text { 엉 }\end{array}$ & 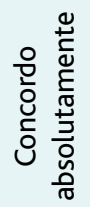 \\
\hline 11 & $\begin{array}{l}\text { Sinto necessidade de ampliar os meus conhecimentos e formação na área da } \\
\text { Nutrição/Alimentação. }\end{array}$ & & & & & \\
\hline 12 & $\begin{array}{l}\text { Para os pacientes obesos, mesmo pequenas perdas de peso ( } 5 \text { a } 10 \% \text { do peso } \\
\text { inicial) podem ser benéficas para a sua saúde. }\end{array}$ & & & & & \\
\hline 13 & $\begin{array}{l}\text { Apenas os pacientes com obesidade grave deveriam ser submetidos a um } \\
\text { tratamento de emagrecimento. }\end{array}$ & & & & & \\
\hline 14 & $\begin{array}{l}\text { O papel do médico de família é referenciar os pacientes obesos a outros } \\
\text { profissionais especializados, em vez de tentar tratá-los. }\end{array}$ & & & & & \\
\hline 15 & Sinto-me competente no diagnóstico e tratamento de pacientes obesos. & & & & & \\
\hline 16 & $\begin{array}{l}\text { Sinto-me capaz de avaliar o estadio de mudança de cada paciente antes de iniciar } \\
\text { um aconselhamento alimentar. }\end{array}$ & & & & & \\
\hline 17 & $\begin{array}{l}\text { É da minha responsabilidade ajudar o paciente a mudar o seu estilo de vida, } \\
\text { apoiando-o e motivando-o. }\end{array}$ & & & & & \\
\hline 18 & $\begin{array}{l}\text { Sinto-me capaz para abordar os benefícios da prática regular de exercício físico c } \\
\text { om os meus pacientes obesos. }\end{array}$ & & & & & \\
\hline 19 & $\begin{array}{l}\text { Sinto-me capaz de elaborar e discutir com os pacientes estratégias para aumentar } \\
\text { a sua confiança e motivação para a mudança de comportamento. }\end{array}$ & & & & & \\
\hline 20 & $\begin{array}{l}\text { Sinto-me capaz de avaliar a ingestão alimentar e a prática de atividade física dos } \\
\text { pacientes obesos. }\end{array}$ & & & & & \\
\hline 21 & A prevenção da obesidade é uma tarefa aborrecida. & & & & & \\
\hline 22 & $\begin{array}{l}\text { Não disponho de tempo suficiente na minha consulta para abordar questões } \\
\text { relacionadas com hábitos alimentares e exercício físico. }\end{array}$ & & & & & \\
\hline 23 & Sinto grandes dificuldades em persuadir os pacientes a mudar de estilo de vida. & & & & & \\
\hline 24 & $\begin{array}{l}\text { Acredito que é importante aconselhar os meus pacientes obesos a perder peso, } \\
\text { mesmo na ausência de outros fatores de risco. }\end{array}$ & & & & & \\
\hline 25 & Sinto-me desconfortável a fazer exame físico a um paciente obeso. & & & & & \\
\hline 26 & $\begin{array}{l}\text { A maioria dos médicos de família não está preparada para aconselhar os seus } \\
\text { pacientes sobre hábitos alimentares e atividade física. }\end{array}$ & & & & & \\
\hline 27 & Sinto necessidade de ter mais formação sobre farmacoterapia da obesidade. & & & & & \\
\hline 28 & $\begin{array}{l}\text { Se devidamente remunerado, estaria disponível a dedicar mais tempo da minha } \\
\text { consulta à gestão e tratamento da obesidade. }\end{array}$ & & & & & \\
\hline
\end{tabular}

